\title{
Fortalezas y debilidades de la educación superior en América Latina para la competitividad global
}

\author{
Santos López-Leyva \\ Facultad de Economía y Relaciones Internacionales. Universidad Autónoma de Baja California. Calzada Universidad no. \\ 14418. Parque Industrial Internacional Tijuana. CP 22390, Tijuana, Baja California, México. \\ (correo-e: sanlop1947@gmail.com; slleyva@uabc.edu.mx)
}

Recibido Ene. 29, 2020; Aceptado Mar. 31, 2020; Versión final Abr. 14, 2020; Publicado Oct. 2020

\section{Resumen}

Este trabajo tiene como objetivo examinar la competitividad de la educación superior en dieciséis países de América Latina con datos del Foro Económico Mundial (WEF, por sus siglas en inglés) desde 2011 a 2017. Se revisan los ocho indicadores del WEF para la educación superior. Se encuentra que existe una correlación entre la competitividad de la educación superior con la competitividad general. La correlación es positiva para catorce países y es negativa en dos de ellos. Se consideran como fortalezas la cobertura, el acceso a internet, la existencia local de investigación, las políticas de formación de profesores y la administración de las universidades. Por otra parte, las debilidades son la calidad del sistema educativo y la enseñanza de matemáticas y ciencias, siendo éste, el indicador de peor comportamiento. Se concluye que la competitividad de la educación superior en América Latina ha observado una mejora considerable en lo que se refiere a la tasa de cobertura.

Palabras clave: competitividad; educación superior; cobertura en educación superior; calidad de la educación superior; políticas en educación superior

\section{Strengths and weaknesses of Latin American higher education for global competitiveness}

\begin{abstract}
The present study aims to examine the competitiveness of higher education in sixteen Latin American countries by analyzing World Economic Forum (WEF) data since 2011 to 2017. Eight WEF higher education indicators are reviewed. The results show that there is a correlation between higher education competitiveness with general competitiveness. The correlation is positive in fourteen countries and negative in two of them. The strengths of higher education competitiveness are found to be coverage, Internet access, local research, teacher training policies, and the quality in the management of universities. On the other hand, the weaknesses are quality of the education and teaching system of mathematics and science, which is the indicator with the worst behavior. It is concluded that higher education competitiveness in Latin American has improved considerably in regard to coverage rate.
\end{abstract}

Keywords: competitiveness; higher education; higher education coverage; higher education quality; higher education policies 


\section{INTRODUCCIÓN}

El objetivo del presente trabajo es exponer las fortalezas y debilidades que presenta la educación superior de América Latina para impulsar la competitividad global de la región. Se utilizan datos del Foro Económico Mundial (WEF, por sus siglas en inglés). Se seleccionaron dieciséis países, por ser éstos los que disponen información completa desde 2011 hasta 2017. Se parte de 2011, porque desde ese año la evaluación de los países se hace utilizando un sistema calificaciones; antes, solo se jerarquizaban por lugares de acuerdo con su nivel competitividad. EI WEF midió la competitividad de los países, hasta 2017, con base en 12 pilares que son: 1) instituciones; 2) infraestructura; 3) comportamiento macroeconómico; 4) educación básica y salud; 5) educación superior y capacitación; 6) eficiencia del mercado de bienes y servicios; 7) eficiencia del mercado laboral; 8) eficiencia del mercado financiero; 9) capacidad de adopción de tecnologías; 10) tamaño del mercado; 11) grado de sofisticación de los negocios y 12) capacidad de innovación. Este artículo se enfoca al análisis del pilar número cinco denominado educación superior y capacitación, el cual se evalúa a través de ocho criterios que son: 1) matrícula en educación secundaria; 2) matrícula en educación superior; 3) calidad del sistema educativo a nivel superior; 4) calidad de la educación en matemáticas y ciencias; 5) calidad de la administración de las universidades; 6) acceso a Internet por parte de las universidades; 7) disponibilidad local de investigación y 8) capacitación del profesorado. La competitividad de este pilar se compara con la competitividad general en cada país.

La educación es importante para el mejoramiento de los procesos de crecimiento y desarrollo económicos de los países, porque además de beneficiar en forma directa a las personas a través de lograr mejores empleos, incrementa los niveles de ingreso y propicia conductas más adecuadas en los individuos; a nivel de la sociedad apoya en crear empleos de mayor calidad, perfecciona el capital humano y forma una ciudadanía más educada y participativa políticamente (De Janvry y Sadoulet, 2016). Si bien la educación influye en múltiples variables del desarrollo económico de un país, dicen Lassibille y Navarro (2004) que, para incidir en el crecimiento y desarrollo económicos, es imprescindible que los sistemas de enseñanza produzcan las cualificaciones que requieren las economías y que, los mercados de trabajo tengan capacidad de incorporar los flujos de titulados que egresan del sistema educativo.

Woessmann (2016) hace una revisión acerca del papel que cumple la educación en diferentes ámbitos de la economía, donde establece cinco dimensiones: 1) educación y prosperidad, ubicando allí los estudios de la productividad y prosperidad tanto a nivel de los individuos y las familias, como en el desarrollo macroeconómico de los países; 2) educación y crecimiento económico, se refiere al papel que desempeña la educación en el crecimiento económico de las naciones en el largo plazo y los costos en que pueden incurrir éstas, tal es el caso de sacrificar su crecimiento como producto de una baja inversión en educación; 3) educación y empleo, con la educación se atiende el problema del desempleo, se influye en el ciclo de vida de los empleos y se busca resolver la disyuntiva entre educación especializada o educación para la formación general; 4) los logros económicos a través de la educación, donde se encuentran las tasas de retorno y las habilidades y capacidades que se forman en los trabajadores; y 5) las políticas educativas, que explican cómo los países formulan agendas de política para el mejoramiento de su sector educativo. Las políticas para impulsar la escolarización de los países han puesto énfasis en la relación entre la formación de capital humano con el crecimiento y el desarrollo económicos; sin embargo, este énfasis se convierte en controversial porque la expansión en la escolarización, no necesariamente, garantiza el mejoramiento de las condiciones económicas de los países. El impacto de las políticas en formación del capital humano se vuelve más fuerte cuando, además de incrementar la cobertura, éstas se enfocan, también, a mejorar la calidad de la educación (Hanushek, 2013)

Existen múltiples estudios acerca del potencial que tiene la educación en los países en vías de desarrollo, desde los que refieren cuestiones macroeoconómicas de la relación de la educación con el crecimiento económico; hasta los enfoques microeocnómicos que utilizan diversas técnicas estadísticas para estimar los retornos privados y sociales de la educación; pasando por la educación para la adopción y difusión de diferentes tecnologías; para reducir las tasas de crecimiento de la población y estudios que ven la educación como un bien con valor intrínseco (Glewwe y Kremer, 2006). El actual modelo de desarrollo global, que se sustenta en la economía basada en el conocimiento, ha dado como resultado el fenómeno de la búsqueda de la competitividad mundial, lo cual se hace como país, pero también se busca mejorar la competitividad de las instituciones de educación superior, proceso que se manifiesta de diferentes formas en los niveles institucional, local, regional, nacional y global (Portnoi et al., 2010).

La educación superior se ha convertido en arena de búsqueda de competitividad en el contexto internacional, pero, además, como dice Lane (2012), el mejoramiento de las instituciones de educación superior es comúnmente usado como un importante recurso para impulsar la competitividad de los países, con base en ello, dichas instituciones han incrementado su potencialidad mediante la inversión en centros y laboratorios, parques científicos y programas de apoyo a las actividades de investigación. Concluye que la moderna universidad de investigación se ha posicionado como un elemento central en el impulso a la competitividad 
de los países. Para Lucchesi (2011), en la base del proceso de desarrollo económico y de la justicia social se encuentra la educación superior. Las universidades entran a conformar los rankings de competencia mundial, por lo que Marginson y Sawir (2006) mencionan que la vieja idea de las universidades integradas a los sistemas nacionales de innovación, proveniente, principalmente, de los pensadores del evolucionismo económico, ha sido minada por el crecimiento en la interdependencia de las naciones y universidades, por el fortalecimiento del flujo mundial de tecnologías, personas, finanzas, idiomas y transmisión de ideas en tiempo real. En los años recientes el crecimiento de la educación superior y los factores de diversificación vienen dados como una respuesta a los mercados competitivos, los cuales están liderados, en mayor medida, por los mercados globales. Otra característica es la movilidad de estudiantes de los países en vías de desarrollo hacia los países desarrollados (Fosket y Maringe, 2012). Pero también se presenta la movilidad de académicos que participan en proyectos de dimensión global.

\section{OTROS ANTECEDENTES}

En el caso de América Latina, Hanushek y Woessmann (2012) señalan que, en los años 60 del siglo XX, esta región experimentaba un significativo crecimiento económico, superaba, como región, a Asia, África Subsahariana, Medio Oriente y Norte de África. En esta misma dirección, McMahon (2008), mediante una función de producción, establece una relación entre educación y desarrollo económico, llegando a la conclusión de que América Latina en las décadas de los 40, 50 y 60 del siglo XX, no lo hizo mal en el impulso a su desarrollo con respecto a los países del este asiático, pero lo problemas empiezan a finales de la década de los años 70s y continúan en los 80 s del mismo siglo. Por otra parte, Christensen et al. (2019) hacen una comparación entre la República de Corea y México, señalan que este último país, en 1960, tenía el doble de ingreso per cápita que la República de Corea, para 1980, era 58\% más rico que Corea y para 2019, el país asiático, era tres veces más rico que México.

La pregunta es porqué Latinoamérica ha tenido un pobre crecimiento en su ingreso per cápita con respecto a esas regiones, generalmente, las respuestas ofrecidas se albergan en factores de productividad, institucionales y financieros, pero Hanushek y Woessmann (2012) opinan que, más bien, este escaso crecimiento es resultado de falta de logros en educación, la cual es el principal componente para el crecimiento de un país en el largo plazo. La región mantiene buenos indicadores en áreas como cobertura y escolaridad esperada, pero no así en la calidad de la educación ofrecida. Generalmente, los estudios de la educación en la región se han realizado con base en el análisis de los años de escolaridad alcanzados, pero queda demostrado que, esta forma de medición presenta múltiples inconsistencias. Para el caso de Latinoamérica, ya desde 2004, Psachoropoulus y Patrinos estimaban que un año más de escolaridad se reflejaba en escaso margen en el incremento en el ingreso, junto con África Subsahariana, eran las regiones donde menor influencia tenía la escolaridad en el ingreso de las personas. Por tal motivo Hanushek y Woessmann (2015) no recomiendan los años de escolaridad como un elemento para la comparación del nivel de educación en el contexto internacional, esto porque un año de escolaridad no es lo mismo para cada país y ponen como ejemplo el nivel de escolaridad alcanzado por Japón y Perú.

Con base en todo lo anterior ha surgido la preocupación por el desarrollo de indicadores que midan el comportamiento de la educación superior de los países en el contexto internacional. Las variables que mayormente se han estudiado son la cobertura y la calidad. Dice Mendoza-Rojas (2015) que la cobertura educativa muestra el esfuerzo que realiza un país para brindar educación a sus habitantes, la cual es medida a través de dos indicadores: la tasa bruta y tasa neta de cobertura. De acuerdo con la Organización de Naciones Unidas para la Educación, la Ciencia y la Cultura (Unesco), la tasa bruta de cobertura (tbc) es la proporción de matrícula total de un nivel determinado, respecto a la población en edad oficial de cursar ese nivel. Existen múltiples factores que influyen en la tasa de matriculación en la educación superior. Declercq y Verboven (2015) mencionan el nivel de ingresos de las familias como un determinante en la matriculación en este nivel educativo, esto por tres razones: a) los estudiantes provenientes de las familias con menores niveles de ingreso presentan mayor sensibilidad a los costos en las colegiaturas; b) estos estudiantes muestran menor nivel de preferencias por la educación superior, ya que tienen necesidad de entrar al mercado de trabajo, y c) generalmente, desarrollan menos habilidades en los niveles educativos previos a los estudios universitarios. Acemoglu y Pischke, desde 2001, habían trabajado el ingreso familiar, educación y raza de los padres como determinantes de la matrícula en educación superior. Por su parte, Didou (2011) señala que, en la discusión de la temática de la cobertura, las relaciones con la búsqueda de la equidad social a través de la educación superior y la promoción de la calidad educativa en este nivel, son dos elementos que han quedado pendientes.

Para 2011, la tasa de cobertura en la región andaba muy cercana al 30\%, con países como Cuba, Argentina y Venezuela que estaban arriba del 50\%. En tanto que para 2016, la Unesco encontró las siguientes tasas de cobertura, México se localizaba diez puntos porcentuales por debajo del promedio de la región de América Latina y el Caribe, que era del $48.4 \%$. En tanto que países como Costa Rica tenía 54\%; Uruaguay, 55.6\%; Colombia, 58.7\%; Argentina, $85.7 \%$, y Chile $90.3 \%$ (ANUIES, 2018). Generalmente, los países que presentan 
baja cobertura, también observan tasas altas de deserción, lo cual, al final lleva a disminuir la tasa de eficiencia terminal. Mendoza Rojas (2015) establece que para México la matrícula en licenciatura y técnico superior universitario ha mantenido un constante crecimiento, pues en 1980 no llegaba al millón de estudiantes y para 2014, se alcanzaron los 3.2 millones; no obstante, las comparaciones internacionales ubican a México por debajo del promedio de América Latina que, para 2012 alcanzó una tasa bruta de cobertura del $43 \%$, once puntos por encima de la de México que fue del $32 \%$, según este autor. Si bien, la educación superior de México ha incrementado su cobertura, otros países latinoamericanos han mostrado un mejor comportamiento en esta variable.

Martin Trow (citado en Rama, 2009) sostiene que la matriculación de la educación superior puede establecerse en tres etapas: élite, masas y universal, según el grado alcanzado por la tasa bruta de matriculación. Si la matrícula no sobrepasa el $15 \%$ es una educación de élite; es de masas si la tasa de matriculación se encuentra entre el $15 \%$ y el $50 \%$; por último, un país cuenta con una matrícula universal si la tasa es mayor al 50\%. Rama (2009) hace una clasificación diferente, estableciendo un acceso de élite si no llega al 15\%; acceso de minorías entre el 15 y el 30\%; de masas hasta el 50\%; universal hasta el $85 \%$ y absoluto, arriba del $85 \%$. Para América Latina resulta más adecuado manejar esta segunda clasificación. Otro elemento que considera el WEF es la calidad de la educación, la cual se observa desde tres dimensiones: a) la calidad del sistema de educación superior; $b$ ) la calidad de los alumnos expresada en la enseñanza de matemáticas y ciencias, y c) calidad en la administración de las instituciones. Existen diversas formas de atender esta variable, la cual se puede ver como logro de mayor prestigio, tanto institucional como personal; como una mejor utilización de los recursos financieros y didácticos; la proporción de profesores con mayores grados académicos; la calidad de los diferentes insumos educativos utilizados en el proceso; la calidad de los contenidos que se imparten; el enfoque de talentos, las personas sobresalientes que se forman y, por último que se denomina el enfoque de calidad total.

Desde principios de los años 90 del siglo pasado, los países de América Latina han impulsado la evaluación con fines de acreditación de instituciones y programas, con lo que se busca incrementar la calidad para facilitar a estudiantes y padres la selección de los programas de calidad, y mejorar los diferentes factores, tales como infraestructura educativa, profesores y prácticas administrativas. La acreditación funciona como una señalización para los diferentes actores relacionados con la educación superior (Haimovich, 2017). Barro y Lee, en 1996, establecieron una función de producción de la calidad de la educación donde los factores considerados son: gasto por estudiante, razón de estudiantes por maestro, salarios estimados de los profesores y duración en la escuela en días por año y horas por día. Aunque no llegan a trabajar con el nivel superior, a nivel secundario la relación de estudiantes por profesor se mantuvo constante de 1960 a 1990 , pero no así el gasto por alumno, que tuvo una fuerte baja, llegando a ser, América Latina, la región con indicador más bajo, después de los países del sur de Asia.

Después de la cobertura y la calidad; el WEF incluye un tercer grupo de indicadores, que se pueden llamar de apoyo, está compuesto por el uso de Internet por parte de las universidades, la disponibilidad de investigación en el ámbito local y la capacitación de los profesores. El acceso a las computadoras en escuelas públicas ha crecido en forma considerable en los últimos 30 años, con datos de la Organización para la Cooperación y Desarrollo Económicos (OECD) (2015) para el año 2012, se tiene por ejemplo que en Colombia había 3.7 estudiantes por computadora; en Chile, 4.7; Perú, 7.9; Uruguay, 8.7; Argentina, 14.1; México, 15.5; Costa Rica, 17.7 y Brasil, 22.1. Un promedio aproximado del $40 \%$ de estas computadoras contaban con acceso a Internet. El porcentaje de estudiantes que tenían al menos una computadora en casa, era el siguiente: Uruguay, 89.6\%; Chile, 88.3\%; Argentina, 83.3\%; Costa Rica, 75\%; Brasil 73.5\%; Colombia, 62.2\%, México, $58.5 \%$ y Perú $52.8 \%$. En este indicador, los países se ubican lejos del promedio de la OECD donde el $95.8 \%$ de los estudiantes cuenta con, al menos, una computadora en casa, pero más lejos de Dinamarca donde el $99.9 \%$ de los estudiantes manifestaron tener una computadora en casa.

Bulman y Fairlie (2016) establecen que no se pueden apreciar grandes impactos en las calificaciones de los alumnos, tanto positivos como negativos con la introducción de las computadoras en las escuelas; sin embargo, señalan que estos impactos son más perceptibles en los países en vías de desarrollo. Existen otros efectos que no son capturados en los exámenes escolares, por ejemplo, para la búsqueda de información, para comunicarse entre sí los estudiantes y con los profesores, atención a factores disciplinarios y de organización de las escuelas, llevar la supervisión de los estudiantes y organizar los contenidos del aprendizaje. Un gran impacto que se puede observar es respecto a la organización de las universidades, sin duda, la informática ha mejorado la administración de las instituciones de educación superior.

La investigación quedó establecida como función sustantiva de las universidades desde la denominada revolución académica, a finales del siglo XIX, a partir de ese tiempo, las instituciones de educación superior, no solo buscan transmitir el conocimiento, sino, favorecer la producción del mismo a través de la investigación. La Comisión Económica para América Latina (CEPAL), tradicionalmente, ha recomendado algunas líneas de investigación que considera prioritarias, entre ellas las relacionadas con el medio ambiente y con la educación. 
Es muy importante la investigación para la utilización y la conservación de los recursos naturales, tales como la pesca, la forestación y los recursos minerales. También es de importancia la investigación en salud, la cual proponga medidas para mejorar las condiciones de bienestar de la población. Investigar acerca de los sistemas educacionales para desarrollar alternativas que fortalezcan las instituciones de educación superior, mejorar los métodos de enseñanza y el establecimiento de mecanismos que fortalezcan la cobertura, la equidad y la calidad de la educación.

En América Latina, sólo el $6 \%$ de los profesores universitarios, en 2002, contaban con el nivel de doctorado, este hecho llevó a las universidades de la región a buscar el mejoramiento de este porcentaje por la vía de implementación de programas doctorales, pero la calidad de estos programas ha sido muy heterogénea, han surgido universidades que no cuentan con las condiciones académicas para la formación de personal en este nivel, sin embargo, se encuentran inmersas en un mercado de formación de docentes (Soto Arango, 2019). Por ejemplo, en México, el $60 \%$ de la matrícula de posgrado se encuentra en instituciones privadas, donde solo se ha logrado acreditar el $2 \%$ de los programas.

En la actualidad, con la responsabilidad de las universidades en la producción de conocimiento se requiere un nuevo perfil académico, que tenga la capacidad para producir conocimiento, a la vez cuente con la habilidad de trasmitirlo, pero con la llamada segunda revolución académica, al profesor se le piden otras habilidades como la de saber relacionarse con el mercado para lograr la comercialización del conocimiento. Galaz Fontes y Gil Antón (2009) establecen algunas tendencias del profesorado universitario en México: 1) la planta académica universitaria ha experimentado un proceso de feminización; 2) el estudio de un posgrado se convierte en la vía de entrada al oficio académico; 3) ha cambiado la edad de incorporación, ahora es más tardía; 4) disponen de mayor capital cultural al provenir de hogares con mayor grado de culturización. De manera frecuente se ha utilizado la "hipótesis del ciclo de vida" para explicar la conexión entre la edad de los profesores con su productividad, de acuerdo con esta teoría, la productividad de un profesor crece en primer lugar por la adquisición de capital humano, la cual declina con la edad, que lleva también al descenso de su productividad.

\section{METODOLOGÍA}

Con datos del WEF, el trabajo revisa la competitividad de cada país, la competitividad del pilar número cinco y los ocho criterios que determinan la competitividad de este pilar, con la finalidad de determinar cuáles son las fortalezas y debilidades de los sistemas de educación superior en América Latina. El análisis se llevó a cabo de la siguiente forma: primero se hizo una comparación entre la competitividad general y la competitividad de la educación superior, con el propósito de revisar el comportamiento de los países; en un segundo momento se analizó la cobertura a través de la tasa bruta de matriculación, tanto a nivel de educación secundaria como en educación terciaria; después, se puso especial énfasis en la calidad de la educación superior en la región, la cual se calificó con la calidad del sistema de educación, la calidad en la enseñanza de matemáticas y ciencias y calidad en las administración de las universidades; al final se revisaron tres elementos que brindan soporte a la educación superior, que son el acceso a Internet de parte de las universidades, la disponibilidad local de investigación y la capacitación del profesorado.

\section{RESULTADOS}

En la Tabla 1 aparece el comportamiento de las calificaciones promedio de cada uno de los indicadores de 2011 a 2017 (Datos del World Economic Forum de 2011-2017). La calificación se fija entre el rango de uno a siete, por lo que se estableció como media 3.5. En cuanto a la competitividad general alcanzó una media de 4, donde Chile es el país con mayor calificación, 4.6, la única variación durante el periodo fue que en 2017 mejoró su calificación a 4.7, el de menor calificación fue Venezuela con 3.3. En educación superior las más altas calificaciones fueron de Chile y Costa Rica quienes alcanzaron una nota de 5, en cambio Nicaragua solo pudo llegar a 3.2. En siete países la competitividad de la educación superior fue mayor que la competitividad general.

En cuanto a la correlación entre competitividad general del país y la educación superior, se encuentran tres grupos de países: a) países donde el índice de correlación es positivo, las dos variables aumentan: Argentina, Chile, Colombia, Costa Rica, República Dominicana, Guatemala, Honduras, México, Nicaragua, Panamá, Paraguay y Uruguay; b) países con coeficiente de correlación positivo, porque las dos variables disminuyen: Brasil y el Salvador, y c) países con coeficiente negativo porque, en el caso de Venezuela aumenta la educación superior y pierde en competitividad general, y Perú, que gana en competitividad general, pero presenta pequeña reducción en educación superior. Entonces, en América Latina existen doce países que han mejorado su competitividad como país, pero también en educación superior; existen dos países que han perdido competitividad en las dos variables. Los que presentan un coeficiente negativo se debe a que la educación superior sigue ganando competitividad en el caso de Venezuela, y para el caso de Perú, el comportamiento mejor se presenta en competitividad general. 
Tabla 1. Calificación promedio de la competitividad general, competitividad de la educación superior y los ocho criterios para medir la competitividad de la educación superior (2011-2017).

\begin{tabular}{|l|c|c|c|c|c|c|c|c|c|c|c|c|c|c|c|c|c|}
\hline País & ARG & BRA & CHL & COL & CRI & DOM & SLV & GTM & HND & MEX & NIC & PAN & PRY & PER & URY & VEN & Promedio \\
\hline $\begin{array}{l}\text { Competitividad } \\
\text { general del país }\end{array}$ & 3.9 & 4.2 & 4.6 & 4.2 & 4.4 & 3.8 & 3.8 & 4 & 3.9 & 4.3 & 3.8 & 4.4 & 3.6 & 4.2 & 4.1 & 3.3 & 4 \\
\hline $\begin{array}{l}\text { Competitividad en } \\
\text { educación superior }\end{array}$ & 4.9 & 4.4 & 5 & 4.4 & 5 & 3.7 & 3.5 & 3.6 & 3.5 & 4 & 3.2 & 4.1 & 3.3 & 4 & 4.6 & 4.4 & 4.1 \\
\hline $\begin{array}{l}\text { Matrícula educación } \\
\text { secundaria }\end{array}$ & 96.5 & 101.8 & 92.5 & 95.9 & 107.7 & 76.7 & 70.8 & 62.7 & 70.8 & 88.8 & 70.4 & 75.5 & 70.5 & 92.4 & 91.2 & 86.8 & 84.4 \\
\hline $\begin{array}{l}\text { Matrícula educación } \\
\text { terciaria }\end{array}$ & 76.5 & 29.7 & 72.7 & 45.6 & 42.1 & 39.9 & 25.9 & 18.6 & 20.4 & 28.8 & 18 & 42.6 & 34.1 & 40.7 & 62.3 & 77.8 & 42.2 \\
\hline $\begin{array}{l}\text { Calidad sistema } \\
\text { educativo }\end{array}$ & 3.3 & 2.8 & 3.5 & 3.4 & 4.7 & 2.5 & 2.8 & 2.6 & 2.9 & 3 & 2.5 & 3.2 & 2.3 & 2.6 & 3 & 2.7 & 3 \\
\hline $\begin{array}{l}\text { Calidad } \\
\text { matemáticas y } \\
\text { ciencias }\end{array}$ & 3.2 & 2.6 & 3.2 & 3.3 & 4.4 & 2.2 & 2.8 & 2.5 & 2.8 & 2.8 & 2.5 & 3 & 2.2 & 2.4 & 3 & 3 & 2.8 \\
\hline $\begin{array}{l}\text { Calidad } \\
\text { administración. } \\
\text { universidades }\end{array}$ & 4.8 & 4.2 & 5.3 & 4.2 & 5.2 & 3.8 & 3.8 & 4.6 & 3.6 & 4.3 & 3.8 & 4 & 3.1 & 4.2 & 4.3 & 4 & 4.2 \\
\hline $\begin{array}{l}\text { Acceso a Internet } \\
\text { de universidades }\end{array}$ & 3.9 & 3.7 & 4.7 & 4 & 4.6 & 3.5 & 3.5 & 3.4 & 3.5 & 3.8 & 2.9 & 4.8 & 2.8 & 3.7 & 5.8 & 3.4 & 3.9 \\
\hline $\begin{array}{l}\text { Disponibilidad } \\
\text { investigación }\end{array}$ & 4.5 & 4.2 & 4.8 & 4.1 & 5 & 4 & 3.9 & 4.6 & 3.9 & 4.4 & 3.3 & 4.3 & 3.1 & 4 & 4.4 & 3.5 & 4.1 \\
\hline $\begin{array}{l}\text { Capacitación } \\
\text { profesorado }\end{array}$ & 3.8 & 4.2 & 4.2 & 3.7 & 4.6 & 3.7 & 3.7 & 4.4 & 4.1 & 3.9 & 3.6 & 4.2 & 3.5 & 3.7 & 3.8 & 3.5 & 3.9 \\
\hline
\end{tabular}

En cuanto a la matrícula en educación secundaria, el WEF maneja la tasa bruta de matriculación, la cual, para el caso de la educación secundaria o su equivalente al nivel 3 en la Clasificación Internacional Normalizada en Educación (ISCED), quien tiene mayor tasa de matriculación es Costa Rica con 107.7\%, le sigue Brasil con $101.82 \%$. Nuestro interés se centra a nivel superior donde el país con mayor cobertura en promedio en el periodo es Venezuela con el $77.8 \%$, le siguen Argentina y Chile con $76.5 \%$ y $72.7 \%$, respectivamente. Al final se encuentran Nicaragua con $18 \%$ y Guatemala con $18.6 \%$. Por tanto, la tasa de cobertura es muy desigual en la región pues, va del $77.8 \%$ de Venezuela al $18 \%$ de Nicaragua, un diferencial de casi 60 puntos. Para 2017, Chile y Argentina mejoran, en forma considerable, para alcanzar tasas del $88.6 \%$ y $82.9 \%$, respectivamente.

Los comportamientos de los indicadores de calidad son: calidad del sistema educativo que alcanza una calificación promedio de 3; educación en matemáticas y ciencias, 2.8; en cambio, la calidad en la administración de las universidades logra una calificación de 4.2, para ser el indicador con mayor calificación. En la calidad del sistema educativo con calificación de 3, sólo Costa Rica (4.7) y Chile (3.5) logran valores iguales o mayores a la media, que es 3.5 , los otros 14 países están por debajo de esa medida, siendo Paraguay el de menor calificación con 2.3. En la enseñanza de matemáticas y ciencias el único país que se ubica por encima de la media es Costa Rica (4.4) y le sigue Colombia con (3.3), existen países que se ubican por debajo de tres, que son Paraguay (2.3), Perú (2.4), República Dominicana y Nicaragua (2.5), Brasil (2.6), el Salvador, Honduras y México (2.8), cabe destacar que 15 países se encuentran por debajo de la media, para ser éste el indicador de peor comportamiento. En calidad de la administración de las universidades, solo Paraguay está por debajo de la media (3.1). En este criterio, la región manifiesta un comportamiento que se equipara con países desarrollados, por ejemplo, en el caso de Chile y Costa Rica alcanzan indicadores semejantes a Japón, Las universidades latinoamericanas manifiestan un buen desempeño en cuanto a adjetiva de gestión y administración.

Por otra parte, en los tres indicadores de soporte se alcanzan calificaciones mayores a la media, el acceso a Internet, con calificación promedio de (3.9); la disponibilidad de investigación, calificación de (4.1) y capacitación del personal, (3.9). En acceso a Internet de parte de las universidades destaca Chile como el país con mayor calificación (4.7), Paraguay se ubica al final con (2.8). En este criterio los países que se ubican por debajo de la media son: Guatemala (3.4), Nicaragua (2.9) y Paraguay (2.8). También en disponibilidad de investigación, Chile aparece en primer lugar y Paraguay al final. En la preparación del profesorado es Costa Rica quien tiene mejor calificación, (4.4) y Paraguay y Venezuela al final (Tabla 1). 


\section{ANÁLISIS Y DISCUSIÓN}

Al llevar a cabo un análisis de la relación entre la competitividad general como país y la competitividad de la educación superior, existen catorce países que presentan una correlación positiva entre estas dos variables; sin embargo, en dos de ellos, estas variables presentan decrecimiento, tales son los casos de Brasil y el Salvador, se encuentran dos países que observan correlación negativa, que son Perú y Venezuela, pero por causas distintas, en el caso del Perú, se ha incrementado la competitividad general y una ligera baja la educación superior; en cambio en Venezuela, la competitividad de la educación superior ha tenido un excelente comportamiento, pero no así la competitividad general, que observa el indicador más bajo en el grupo de países.

De manera general, se advierte que la competitividad general y de la educación superior presentan comportamientos similares, lo cual demuestra relación entre estas dos variables. Existen países que muestran correlaciones mayores a 0.6, tales son los casos de República Dominicana, 0.94; Guatemala, 0.88; Costa Rica, 0.66; Argentina, Honduras y México, 0.6. Entre 0.5 y menores a 0.6, se encuentran Colombia 0.51; el Salvador, 0.58; Nicaragua y Paraguay 0.57; entonces, diez países presentan una correlación fuerte al obtener valores mayores a 0.5 . Aunque con coeficiente muy pequeño, pero positivo, se encuentra Chile con 0.04 , que manifiesta crecimiento significativo de la competitividad de la educación superior, pero un ligero crecimiento en la competitividad general. Con todos estos casos se concluye con algo similar a lo señalado por Lassibille y Navarro (2004), quienes observan que no sólo es necesario mejorar la competitividad de la educación, sino el ambiente donde se desenvuelve la misma. Para que la educación superior tenga un buen efecto en el crecimiento del país, es necesario un desempeño adecuado en otros pilares, pero sobre todo un buen comportamiento institucional. También, se mantiene lo que Portnoi et al. (2010) opinan en torno a que los países deben mejorar la competitividad en las dos direcciones. En cambio, Hanushek y Woessmann (2012) opinan que los países deben impulsar el mejoramiento de la educación superior porque es una variable que influye en la competitividad general de un país en el largo plazo.

Se cuenta con dos países donde la competitividad general y la educación superior disminuyen, tales son los casos de Brasil y el Salvador. En referencia a Brasil, aunque la caída de la educación superior es leve, existen otras variables que empujan la competitividad general a la baja como el comportamiento de las instituciones, donde en la confianza en los políticos, es el país que aparece en último lugar a nivel global; otros indicadores de mal desempeño son la carga en las regulaciones del gobierno y el manejo de los fondos públicos. En el caso de el Salvador, también presenta su peor comportamiento en el campo de las instituciones, ya que sólo cuatro países de los 137 seleccionados por el WEF, tienen inferior comportamiento que esta nación. Los indicadores con mayor deficiencia se encuentran en los efectos del crimen organizado, la confianza en los políticos y la eficiencia en el manejo del gasto del gobierno, que junto a los indicadores referidos a la innovación presentan un mal comportamiento.

Al realizar un análisis por indicadores de educación superior, se encuentra que la cobertura se ha incrementado, pues como señala Schwartzman (2008), la tasa neta de matriculación en 2003, en Argentina era del $60 \%, 22.7 \%$ en Brasil, $46.2 \%$ en Chile y $23 \%$ en México, el promedio en la región era del $27 \%$, lejos del $69 \%$ de Europa Occidental y Norteamérica y del 51\% de Europa Central. Para 2017, Argentina alcanzó una tasa del $82.9 \%$, Brasil del $50.6 \%$, Chile del $88.6 \%$ y México casi $30 \%$. Aquí hay que marcar el gran salto de Chile y Brasil. La tasa de la región se incrementó hasta lograr un $47 \%$, lo cual implica un crecimiento de 20 puntos porcentuales. Lo que no se observa es un proceso de convergencia, pues de Chile a Nicaragua hay una diferencia de 70 puntos, es decir, la brecha se ensancha. Este crecimiento manifiesta que varios países tienen tasas similares y hasta superiores de cobertura a los países desarrollados, por ejemplo, en 2017, Alemania tuvo 68.3\%, Japón 63.4\% y Estados Unidos 85.8\%.

La capacidad que manifiestan los países para atender su matriculación ha llevado a mejorar el promedio de escolaridad esperada en América Latina, para 2017 se tenía a Argentina y Chile con tasas de escolaridad esperada de 17.3 y 16.3 años, respectivamente, que superaban la escolaridad esperada de la media de los 51 países de más alto desarrollo que era de 16 años, según el índice de Desarrollo Humano; donde la media de América Latina casi alcanzaba los 14 años, ubicándose por debajo de los países de más alto desarrollo por más de años. En cambio, el nivel medio de escolaridad, Argentina y Chile, las tasas más altas, alcanzaban 9.9 años y el promedio de los 51 países de mayor desarrollo era de 11.5 años.

En este indicador América Latina logró 8.1 años. Aquí se observaba un diferencial de 3.4 años entre la media de escolaridad de América Latina y los países de mayor desarrollo. Esto indica que la región, a pesar de contar con tasa de escolaridad baja, las expectativas de la escolaridad esperada son altas, lo cual es influencia del incremento en la cobertura en educación. Un elemento que influye en la cobertura es, como establecen Declerq y Verboven (2015), el nivel de ingreso, aquí se observa que los países que presentan mayor nivel ingreso per cápita, son los que presentan mayor cobertura en educación superior. 
Considerando la distribución que propone Rama (2009), existen cinco países con acceso de minorías a la educación superior, pues su tasa de cobertura para 2017, no llegaba al 30\%, estos países son Nicaragua, 18\%; Guatemala, 21.8\%, Honduras, 22.1\%; el Salvador, 29.1\%, y México, 29.9\%. Con acceso de masas, son tres países los que se encuentran en el rango de 30\% al 50\%: Paraguay, 35.1\%; Panamá, 38.7\%, y Perú, 40.5\%. En el rango de 50\% al $85 \%$, que corresponde a países con acceso universal se ubican: República Dominicana, 50.1\%; Brasil, 50.6\%; Uruguay, 55.6\%; Colombia y Costa Rica con 55.7\%; Venezuela, 77\%, y Argentina, $82.9 \%$. Por último, en el rango de acceso absoluto, con una tasa superior al $85 \%$, se encuentra Chile con $88.6 \%$.

En cuanto a la búsqueda de la calidad, en América Latina se ha seguido un modelo que insiste más en la evaluación de procesos que en resultados, eso implica, en parte, que haya mejorado en forma considerable la calidad en la administración de las universidades, porque en este indicador alcanza el (4.2) en promedio, donde Chile logra calificación de (5.3), Costa Rica (5.2) y Argentina (4.8), cifras que se comparan con los países de mayor desarrollo, por ejemplo: Estados Unidos (5.9), Japón (4.3) y Alemania (5.3). No todos los países presentan calificaciones altas por lo que se observa una desviación estándar del 0.57 , que es muy superior con respecto a otros criterios. En un ejercicio de comparación, utilizando los indicadores del pilar 5 del WEF, entre cuatro países de América Latina: Argentina, Brasil. Chile y México, con cuatro países de Asia: Japón, Hong Kong, Singapur y Corea de Sur, se encontró que el indicador más similar entre estos dos grupos es la calidad en la administración y gestión de las universidades (López Leyva y Rhoades, 2016).

En calidad del sistema de educación superior, Costa Rica es el país que alcanza la mayor calificación promedio con 4.4 y en 2017 estaba en el lugar número 27 en el ranking mundial del WEF. Chile alcanza una calificación promedio de 3.5, los restantes países se ubican por debajo de la media, con Paraguay al final con calificación de 2.3 y en el lugar 135 del ranking mundial, sólo dos países de este ranking presentan un comportamiento peor que Paraguay. Existe un rango entre Costa Rica y Paraguay de 108 lugares y una diferencia mayor a dos puntos en sus calificaciones. En conclusión, la calidad del sistema de educación superior latinoamericano es baja, con grandes diferencias entre los países. Esta baja calidad de los sistemas de educación superior se manifiesta también en el reducido número de universidades de clase mundial que aparecen en los diferentes listados de organismos que evalúan universidades a nivel mundial, por ejemplo, en el Academic Ranking World Universities, solo aparecían nueve universidades latinoamericanas entre las primeras 500 en 2019.

En la enseñanza de matemáticas y ciencias, Costa Rica es el único país que pasa la calificación media, pues logra, en promedio (4.4), y en 2017 apareció en el lugar 52 del ranking mundial; el país con menor calificación, es también Paraguay que apenas logró (2.2), para ocupar el lugar 136 a nivel global, solo un país tuvo peor comportamiento que Paraguay. Quince países se ubican por debajo de la calificación media. La mayoría de países presentan una calificación baja y con cierta similitud, lo cual hace que este indicador observe la menor desviación estándar de apenas 0.14 . Sin duda, la enseñanza de las matemáticas y ciencias constituye un gran problema para la educación en América Latina, lo cual también se muestra en el comportamiento de los resultados del Programa Internacional para la Evaluación de Estudiantes (PISA, por sus siglas en inglés). Otro intento por medir las habilidades en este campo es la prueba International Student Achievement (ISATs), donde Hanushek y Woessmann (2015) incluyen los puntajes para Brasil, Chile, Colombia, México, Perú y Uruguay.

Glewwe y Kremer (2006) señalan que en matemáticas existe una diferencia de tres años entre alumnos de países desarrollados con respecto a estudiantes de países en vías de desarrollo; además, el porcentaje de estudiantes con muy bajas habilidades es muy alto en estos últimos países, por ejemplo, en Argentina era del 22.6\%; Perú $54.1 \%$ y Brasil 23.3\%, estos autores toman datos de la prueba PISA aplicada en el año 2000. Pero considerando PISA 2018, en matemáticas se evaluaron 78 países, China se encuentra en primer lugar con 591 puntos, el país latinoamericano que alcanzó mayor puntaje fue Chile con 417 puntos, lo cual implica que hay una diferencia de 174 puntos entre ambos países; pero se observa una diferencia de 266 puntos de parte de República Dominicana que alcanza 325 puntos, con respecto al primer lugar. Esto implica que un estudiante dominicano de 15 años, apenas domina el 55\% de los contenidos manejados por un estudiante chino de la misma edad.

En el tercer grupo de indicadores está compuesto por los elementos de soporte a la educación superior donde se encuentra el acceso a Internet por parte de las universidades, en el cual la región alcanza la calificación promedio de (3.9), con Uruguay en el primer lugar logrando una calificación de (5.8), que permite ubicarse en el lugar número 20 en el ranking mundial de países. En el extremo opuesto se encuentran Paraguay y Nicaragua, con calificaciones de (2.8) y (2.9) respectivamente, ubicados en los lugares 123 y 117 del mismo ranking en 2017. La distancia entre Uruguay y Paraguay es de 3 puntos y en ranking mundial muestra una diferencia de 103 lugares. Micheli y Armendáriz (2011), al estudiar una muestra de 41 universidades latinoamericanas, concluyen que las estructuras virtuales de educación en estas instituciones son de creación reciente y no una actividad consolidada; además se puede observar la carencia de personal preparado en el 
uso de este tipo de tecnologías. En la disponibilidad local de investigación, también Costa Rica se ubica en el primer lugar del grupo, con cinco puntos de calificación y en el lugar 21 del ranking global. En este indicador también se rebasa la calificación media que logra un promedio de 4.1 de calificación.

Sin embargo, es el indicador que presenta la mayor desviación estándar que es igual 0.7, esto por las diferencias en las estructuras de investigación que existen entre los países. Aquí también Paraguay se ubica al final con (3.1) de calificación y en el lugar 128 del ranking mundial. Es pertinente resaltar la diferencia de 107 lugares en el contexto mundial entre Costa Rica y Paraguay. Para Lucchesi (2011), en la actualidad, la universidad configura un nuevo paradigma donde la investigación es la propia enseñanza, o sea docencia e investigación no son dos funciones separadas. En cuanto a la capacitación del profesorado, también Costa Rica aparece en primer lugar con calificación de (4.6), y en el lugar 37 del ranking de países. Al final aparecen Venezuela y Paraguay con una calificación de (3.5). En este indicador, los países estudiados también sobrepasan la calificación media de (3.5).

Uno de los elementos que se puede observar en los datos anteriores es el rango de diferencia elevado que existe entre los lugares que ocupan los países en el ranking del WEF, pues al revisar los diferentes indicadores se encuentra que: en calidad del sistema educativo, en 2017, Costa Rica apareció en el lugar 27, en tanto que Paraguay se fue hasta el 135, una diferencia de 108 lugares; mientras que, en calidad en matemáticas y ciencias, Costa Rica estaba en el lugar 52 y Paraguay en el 136. En administración de las universidades, Costa Rica en el 21 y Paraguay en el 126, un diferencial de 105 lugares. Como se advierte, en el campo de la calidad, el dominio lo tiene Costa Rica, en tanto que al final de los 16 países aparece Paraguay. En el uso de Internet en las universidades, el mejor comportamiento es de Uruguay, que ocupa, internacionalmente, el lugar número 20 y Paraguay el 117. Disponibilidad de investigación, Costa Rica en el lugar 21 y Paraguay en el 120; por último, la capacitación del profesorado, Costa Rica en el 37 y Paraguay en el 115. Las mayores distancias se encuentran en calidad del sistema educativo entre Costa Rica y Paraguay, con una diferencia de 108 lugares y en disponibilidad de investigación, entre los mismos países, con 107 lugares. La pregunta obligada es qué hace esta diferencia entre estos dos países con casi el mismo tamaño de población y situados en la región latinoamericana. Para 2018, Costa Rica tenía 5 millones de habitantes y Paraguay 7.1, pero con un diferencial 5845.6 dólares anuales a favor del primero en el ingreso per cápita, los otros diferenciales se ubican en el desempeño institucional y en la capacidad de innovación.

La variable que presenta mayor influencia en la competitividad de la educación superior es la calidad, pues los tres indicadores de calidad son los que manifiestan una correlación más alta con la competitividad de la educación superior, calidad en la administración de las universidades presenta un coeficiente de 0.81 , y la calidad del sistema educativo y calidad en la enseñanza de matemáticas y ciencias alcanzan un coeficiente del 0.7. Así la calidad en la administración de las universidades es el indicador que cubre mayor calificación, a la vez, es el que tiene la mayor correlación con la competitividad de las universidades. Entonces, si se quiere mejorar la posición de la educación superior de América Latina en el contexto mundial, es necesario mejorar su calidad en el sistema y en matemáticas y ciencias. En la región se han implementado una serie de programas y organismos para el impulso de la calidad de la educación superior, solo por mencionar algunos ejemplos, en México se tienen, los Comités Interinstitucionales de Evaluación de la Educación Superior (CIEES), creados en 1991, los cuales otorgan mucha importancia a la gestión institucional; el Consejo para la Acreditación de la Educación Superior A. C. (Copaes A.C.), creado en el año 2000. En Colombia, el Consejo Nacional de Acreditación (CNA) y la Comisión Nacional de Aseguramiento de la Calidad (Conoces), entre otros. En Argentina se encuentra la Comisión Nacional de Evaluación y Acreditación Universitaria (Coneau), y así en los diferentes países se crearon organismos para esta finalidad. Lo anterior manifiesta la preocupación en la región por la institucionalización de las actividades de evaluación y acreditación de la educación superior.

Al hacer una comparación de los indicadores de los países con la media de 3.5, se encuentra que, en calidad del sistema de educación superior, solo un país rebasa esta medida, lo mismo sucede con enseñanza de matemáticas y ciencias, por tal motivo, estas dos dimensiones son las de mayor debilidad en la educación superior de América Latina. Aquí la buena noticia es que los organismos latinoamericanos relacionados con el mejoramiento de la educación, han asumido compromisos con el mejoramiento de la calidad, tal como se establece en el objetivo cuatro de los Objetivos del Desarrollo Sostenible, esperamos buenos resultados.

La región latinoamericana debe incrementar su participación en exámenes y pruebas internacionales con la finalidad de mejorar su posición en la competitividad a nivel internacional, hasta la fecha su participación ha sido reducida y son pocos países los que participan, Hanushek y Woessmann (2015) señalan que existen 36 diferentes pruebas para evaluar las habilidades de los estudiantes en matemáticas, ciencias y capacidades lectoras, donde, desde 1964 a 2003, solo siete países latinoamericanos habían participado en algunas de estas pruebas, estos países: Argentina, Brasil, Chile, Colombia, México, Perú y Uruguay. Antes del año 2000, solo Chile y Colombia había participado en Evaluation of Educational Achievement (IEA), programa de la OECD en matemáticas y ciencias. Para mejorar la posición en la competitividad de la educación superior de 
América Latina es necesario que la región participe, de manera más intensiva, en las pruebas de evaluación de las habilidades de los estudiantes en el contexto internacional. En general, la educación superior de la región debe atender de mejor manera las evaluaciones internacionales de las diferentes funciones de la educación. Pero, no solamente participar, sino también impulsar las políticas que llevan a realizar una incursión con mayor éxito en este tipo de evaluaciones y, por tanto, en la competitividad internacional.

\section{DISCUSIÓN FINAL}

América Latina, en el periodo estudiado, ha mejorado su competitividad en la educación superior, pero esta mejora ha sido muy desigual; los países que muestran avances considerables como Costa Rica y Chile se van separando, cada vez más, de los países que casi permanecen estáticos, tales son los casos de el Salvador, Honduras, Nicaragua y Paraguay, entonces, se observa un proceso de divergencia en la competitividad de la educación superior entre los países de la región. De los dieciséis paises, trece de ellos han mejorado su competitividad de educación superior, Brasil y el Salvador bajaron en su competitividad general y en educación superior, Perú observó ligera baja en educación superior y Venezuela mejoró en educación superior, pero no en competitividad en general. Entonces doce países muestran mejoarmiento en la competitvidad de ambas variables.

La región ha observado un gran salto en la tasa bruta de matriculación al pasar del $27 \%$ en 2003, al $47 \%$ en 2017, con los casos destacados de Chile, Brasil y Argentina. También es significativo el aumento en la tasa de escolaridad esperada, donde Argentina y Chile se encuentran a nivel de los países desarrollados en el comportamiento de este indicador. Pero no se puede decir lo mismo en la tasa de escolaridad media, donde la región apenas sobrepasa los ocho años. Se incrementó a siete el número de países con matrícula de acceso universal, Chile alcanzó lo que se denomina matrícula de acceso absoluto. Este incremento en la tasa de matriculación ha mejorado la escolaridad esperada, pero manifiesta bajo impacto en escolaridad media. El incremento en la cobertura constituye una fortaleza en la educación superior de la región.

En el campo de la calidad en educación superior, un indicador de significancia es la calidad en la administración de las universidades, siendo éste, el criterio de mejor comportamiento y que también, en algunos países latinoamericanos, se asemeja a la ruta que siguen los países desarrollados. Esto es resultado de los procesos de evaluación impulsados en la región, los cuales están centrados en la administración y gestión de las universidades. Algunos países como Argentina, Colombia y México han desarrollado amplios marcos de referencia para la evaluación de la educación superior, pero con sus estándares e indicadores se persigue, centralmente, el mejoramiento de las funciones de administración y gestión de las instituciones.

La gran debilidad en la región es la enseñanza de matemáticas y ciencias, esto se observa no sólo en el indicador del WEF, sino también en la aplicación de la prueba PISA y en los pocos resultados que se tienen del Programa para Evaluación Internacional de Competencias de los Adultos (PIAAC). América Latina, tiene la urgencia de avanzar en el mejoramiento en estas áreas de conocimiento. Otra debilidad es la baja calificación alcanzada por el sistema de educación superior, los países estudiados tienen la necesidad de impulsar políticas para mejorar los indicadores de calidad de este sistema, pero, principalmente, en los contenidos académicos y en la enseñanza de dichos contenidos. Un camino es instrumentar las evaluaciones en torno a las funciones sustantivas de la universidad y el impulso a procesos de capacitación del profesorado. Los organismos latinoamericanos relacionados con la calidad asumen, de forma constante, el mejoramiento de la educación superior en la región, pero esto no puede ser posible si este compromiso no se contrae, de manera plena, por parte de los diferentes actores que participan en el proceso.

En los tres restantes indicadores que son acceso a Internet por parte de las universidades, disponibilidad local de investigación y capacitación del profesorado, donde el mejor evaluado es la disponibilidad de investigación local, se observa que sobrepasan la media de 3.5. Aunque el acceso a Internet y la capacitación de profesores alcanzan la misma puntuación, resulta de mayor importancia poner atención a la capacitación del profesorado para la mejora el sistema de educación superior. Estas constituyen fortalezas de la educación superior de la región. Se consideran como debilidades del sistema de educación superior en América Latina, la calidad del sistema de educación superior y la calidad en la enseñanza de las matemáticas y ciencias. Como fortalezas, entonces, son el incremento en la matrícula, la calidad de la administración de las universidades y los mecanismos de soporte como son el acceso a Internet, la existencia de investigación local y la capacitación de los profesores.

\section{CONCLUSIONES}

Basado en el estudio realizado, los resultados y su discusión se pueden extraer las siguientes conclusiones principales:

1.-La competitividad de la educación superior en América Latina ha observado una mejora considerable en lo que se refiere a la tasa de cobertura, lo cual ha posiblitado, también, que algunos países alcancen promedios altos en la esperanza educativa, incluso, logran tasas similares a los países desarrollados. Pero en este 
indicador se observa un gran diferencial porque existen países con acceso de minorías, que no llegan al 30\%; pero otros se ubican en el rango de acceso universal, arriba del $50 \%$ y hasta de acceso absoluto mayor al $85 \%$.

2.- La preocupación por el mejoramiento de la calidad se ha centrado en las funciones de administración y gestión de las instituciones, lo cual ha permitido que algunas instituciones alcancen, en esta función, una competitividad similar a las instituciones de los países desarrollados; pero no pasa lo mismo con la calidad del sistema educativo y en enseñanza de matemáticas y ciencias donde la competitividad de nuestros países es baja. En cuanto a matemáticas, las instituciones no participan en muchas de las pruebas que existen en el contexto internacional. Se puede decir que se ha mejorado en una competitividad blanda, que es la gestión y la administración; pero muy poco se ha avanzado en una competividad sólida que es la enseñanza y dominio de conocimientos en los campos de las matemáticas y ciencias.

3.- Se observan tendencias similares en la mayoría de los países en cuanto al comportamiento de la competitividad de la educación superior con la competitividad general de los países, lo cual arroja coeficientes de correlación positivos en la mayoría de los casos.

4. Al llevar a cabo una comparación entre países, es posible observar diferencias muy marcadas en los diferentes indicadores, esto se observa con mayor precisión si la comparación se hace utilizando los lugares que ocupan los países en el ranking. Por ejemplo, existe una diferencia de 108 puntos entre Costa Rica y Paraguay en el indicador de calidad del sistema, si en 2017 se evaluaron 137 países, este diferencial es muy amplio, lo cual indica alta heterogeneidad en el comprtamiento de este criterio.

5. Los criterios que alcanzaron mayor puntuación fueron la calidad de la administración y gestion y la disponibilidad de investigación local, pero a la vez fueron los indicadores que tuvieron una mayor desviación estándar, esto se debió a que los países no mejoraron de forma unánime, ya que no todos los países presentaron avances notables en este indicador. Por el contrario, el peor comportamiento estuvo en enseñanza de matemáticas y ciencias, pero aquí se observó una desviación pequeña ya que la mayoría de los países alcanzaron valores bajos.

\section{REFERENCIAS}

Acemoglu, D., y Pischke, J. S., Changes in the Wage Structure, Family Income and Children's Education, doi:10.1016/S0014- 2921(01)00115-5, European Economic Review, 45 (4-5), 890-904 (2001)

Asociación de Universidades e Instituciones de Educación Superior (ANUIES), Visión y Acción 2030. Propuesta de la ANUIES para Renovar la Educación Superior en México, 1a. ed. pp. 55-77, ANUIES, Ciudad de México, México, (2018)

Barro, R. J. y Lee, J.W., International Measures of Schooling Years and Schooling Quality, The American Economic Review, 86 (2), 218-223 (1996)

Bulman, G. y Fairlie, R. W., Technology and Education: Computers, Software, and the Internet, in Handbooks in Economics. Economics of Education, 1a. ed. Elsevier-NH, v. 5, pp. 239-280, Amsterdam, Netherlands, (2016)

Christensen, C. M., Ojomo, E. y Dillon, K., The Prosperity Paradox. How Innovation Can Lift Nations out of Poverty, 1a., 155-178, Harper Business, New York, USA, (2019)

Declercp. K. y Verboven F., Socio-economic Status and Enrollment in Higher Education: Do Costs Matter? http://dx.doi.org/10.1080/09645292.2015.1047822, Education Economics, 23 (5), 532-556 (2015).

De Janvry, A. y Sadoulet, E., Development Economics. Theory and Practice, 1a. ed., pp. 593-624, Routledge, London, England, (2016).

Didou Aupetit, S., Cobertura y Promoción de la Equidad en el Sistema de Educación Superior en México, ISSN $0185-$ 2698, Revista Perfiles Educativos, XXXIII (número especial), 59-65, (2011).

Foskett, N. y Maringe, F. The Internationalization of Higher Education, in Globalization and Internationalization in Higher Education. Theoretical, Strategic and Management Perspectives.1a.ed. Continuum, pp. 1-13, London, England, (2012).

Galaz Fontes, J. F. y Gil Antón, M., La Profesión Académica en México: Un Oficio en Proceso de Reconfiguración, ISSN 1607-4041, Revista Electrónica de Investigación Educativa, 11(2), 1-32 (2009).

Glewwe, P. y Kremer, M., Schools, Teachers, and Education Outcomes in Developing Countries, in Handbooks in Economics. Handbook of the Economics of Education, 1a. ed., Elsevier-NH, V. 2, pp. 946-1017, Amsterdam, Netherlands, (2006).

Hanushek, E. A., Economic Growth in Developing Countries: The Role of Human Capital, Economics of Education Review, 37, 204-212 (2013).

Hanushek, E. A. y Woessmann, L., The Knowledge Capital of Nations, 1a. ed. pp. 9-65, CESifo-The MIT Press, Cambridge MA, USA, (2015).

Hanushek, E. A. y Woessmann, L., Schooling, Educational Achievement, and the Latin American Growth Puzzle, https://doi.org/10.1016/j.jdeveco.2012.06.004, Journal of Development Economics, 99,497-512 (2012) 
Haimovich Paz, F. Equity, Quality, and Variety of Higher Education, in At a Crossroads. Higher Education in Latin America and the Caribbean, 1a. ed. World Bank Group, pp. 77-113. Washington DC, USA, (2017)

Lane, J. E. Higher Education and Economic Competitiveness, in Universities and colleges as Economic Drivers. Measuring Higher Education's Role in Economic Development, 1a. ed., SUNY Press, pp. 1-30, New York, e USA, (2012).

Lassibille, G. y Navarro Gómez M. L., Manual de Economía de la Educación. Teoría y Casos Prácticos, 1ª . ed., pp.145179, Ediciones Pirámide, Madrid, España, (2004)

López-Leyva, S. y Rhoades, G., Country Competitiveness Relationship with Higher Education Indicators, http://dx.doi.org/10.4067/S0718-27242016000400007, Journal of Technology Management \& Innovation, 11 (4), 47-55 (2016).

Lucchesi, M. A. S., La Universidad Internacional en América Latina: un Nuevo Paradigma para el Siglo XXI, doi: 10.4067/S0718-50062011000100005, Revista Formación Universitaria, 4 (1), 25-36 (2011)

Marginson, S. y Sawir, E., University Leaders' Strategies in the Global Environment: A Comparative Study of Universitas Indonesia and Australian National University, https://doi.org/10.1007/s10734-004-5591-6, Higher Education, 52(2), 343$373(2006)$

McMahon, W. W. Education and Development. Measuring the Social Benefits, 1a. ed. pp. 19-67, Oxford University Press. Oxford, England, (2008).

Mendoza-Rojas, J., Ampliación de la Oferta de Educación Superior en México y Creación de Instituciones Públicas en el Periodo 2001-2012, https://doi.org/10.1016/j.rides.2015.04.001, Revista Iberoamericana de la Educación Superior, VI (16), 3-32 (2015)

Micheli, J. y Armendáriz, S., Estructuras de Educación Virtual en la Organización Universitaria. Un Acercamiento a la Sociedad del Conocimiento, doi: 10.4067/S0718-50062011000600005, Revista Formación Universitaria, 4(6), 35-48 (2011).

OECD (2015), Students, Computers and Learning: Making the Connection, http://dx.doi.org/10.1787/9789264239555-en, 1a. ed. 15-37, OECD Publishing, Paris, France, (2015).

Portnoi, L. M., Bagley, S. y Rust, D., Mapping the Terrain: the Global Competition Phenome in Higher Education, in Higher Education Policy, and the Global Competition Phenome, 1a. ed., Palgrave Macmillan, pp. 1-13, , New York, USA, (2010)

Psacharopoulus, G. y Patrinos, H. A., Returns to Investment in Education: A Further Update, https://doi.org/10.1080/0964529042000239140, Education Economics, 12 (2), 111-134 (2004).

Rama, C., La Tendencia a la Masificación de la Cobertura de la Educación Superior en América Latina, ISSNe:1681-5653, Revista Iberoamericana de Educación, 50,173-195 (2009)

Schwartzman, S. The Leading Latin American Universities and the Contribution to Sustainable Development in the Region, in University and Development in Latin America, 1a. ed., Sense Publishers, pp. 5-21, Rotterdam, Netherlands, (2008).

Soto Arango, D., El Profesor Universitario de América Latina. Hacia una Responsabilidad Ética, Científica y Social, revistas.uptc.edu.co/revistas/index.php/historia_educacion_latinamerican/article/view/1539/1535, ISSN: 2256-5248 Revista Historia de la Educación Latinoamericana 13, 166-188 (2009).

Woessmann, L., The Economic Case for Education, http://dx.doi.org/10.1080/09645292.2015.1059801,Education Economics, 24(1), 3-32 (2016) 